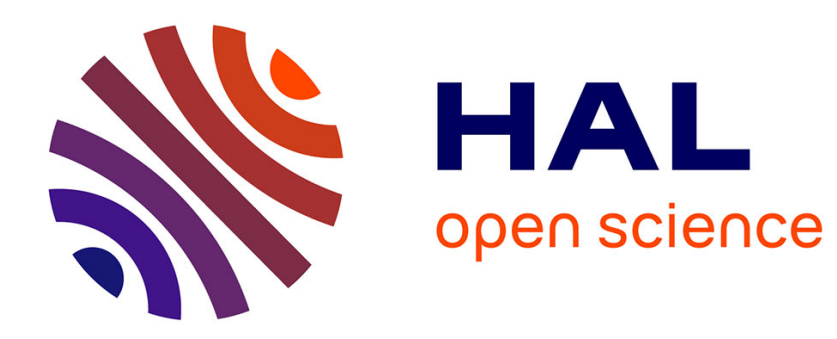

\title{
Moffatt-type flows in a trihedral cone
}

Julian F. Scott

\section{- To cite this version:}

Julian F. Scott. Moffatt-type flows in a trihedral cone. Journal of Fluid Mechanics, 2013, 725, pp.446-461. 10.1017/jfm.2013.180 . hal-00931496

\section{HAL Id: hal-00931496 https://hal.science/hal-00931496}

Submitted on 17 Jun 2014

HAL is a multi-disciplinary open access archive for the deposit and dissemination of scientific research documents, whether they are published or not. The documents may come from teaching and research institutions in France or abroad, or from public or private research centers.
L'archive ouverte pluridisciplinaire HAL, est destinée au dépôt et à la diffusion de documents scientifiques de niveau recherche, publiés ou non, émanant des établissements d'enseignement et de recherche français ou étrangers, des laboratoires publics ou privés. 


\title{
Moffatt-type flows in a trihedral cone
}

\author{
Julian F. Scott $†$ \\ LMFA, Université de Lyon, Ecole Centrale de Lyon, Ecully 69134, France \\ (Received 9 March 2013; revised 9 March 2013; accepted 29 March 2013; \\ first published online 14 May 2013)
}

The three-dimensional analogue of Moffatt eddies is derived for a corner formed by the intersection of three orthogonal planes. The complex exponents of the first few modes are determined and the flows resulting from the primary modes (those which decay least rapidly as the apex is approached and, hence, should dominate the near-apex flow) examined in detail. There are two independent primary modes, one symmetric, the other antisymmetric, with respect to reflection in one of the symmetry planes of the cone. Any linear combination of these modes yields a possible primary flow. Thus, there is not one, but a two-parameter family of such flows. The particletrajectory equations are integrated numerically to determine the streamlines of primary flows. Three special cases in which the flow is antisymmetric under reflection lead to closed streamlines. However, for all other cases, the streamlines are not closed and quasi-periodic limiting trajectories are approached when the trajectory equations are integrated either forwards or backwards in time. A generic streamline follows the backward-time trajectory in from infinity, undergoes a transient phase in which particle motion is no longer quasi-periodic, before being thrown back out to infinity along the forward-time trajectory.

Key words: low-Reynolds-number flows, Stokesian dynamics

\section{Introduction}

Following the intriguing discovery by Moffatt (1964) of an infinite log-periodic sequence of eddies in two-dimensional corner flow, a number of studies (see Shankar 2007, chapter 11, and references therein) have aimed at three-dimensional extensions. Two types of problems can be distinguished: those which are three-dimensional versions of the Taylor paint scraper problem, in which the flow is driven by wall motion extending to the corner, and those which, like the Moffatt problem, have no wall motion near the corner. An example of the former type is Hills \& Moffatt (2000).

The second class of problems includes the one studied in this paper. As in the twodimensional case, it is argued that the effective Reynolds number decreases to zero as the corner is approached, so a description using Stokes flow is appropriate in the nearcorner region. Cases which have been successfully treated include three-dimensional flow in a two-dimensional corner (see e.g. Moffatt \& Mak (1998), who showed that eddies need not occur for three-dimensional flow), the circular cone (Wakiya 1976; Liu \& Joseph 1978; Malyuga 2005; Shankar 2005) and two concentric circular cones (Malhotra, Weidman \& Davis 2005; Hall, Hills \& Gilbert 2009). 
There is, however, an interesting example which is conspicuous by its absence, namely the corner formed by the intersection of three planes, a geometry referred to as a trihedral cone. This might, for instance, represent flow near the corner of a rectangular container. To quote Shankar $(2007, \S 11.3)$, 'Nothing is known about such flows...'. To the best of the author's knowledge, this remains the case today; hence, the present paper, which provides the first analysis of such a flow. We study the analogue of Moffatt eddies in a corner bounded by three orthogonal planes. This geometry has previously been used by, e.g. Gomilko, Malyuga \& Meleshko (2003), but with forcing by wall motion extending to the apex, rather than driving by distant flow. As noted above, this is a three-dimensional version of the Taylor paint-scraper problem, rather than the Moffatt one, and has nothing to say about the near-apex asymptotics of flow in the absence of wall motion which we study here.

Section 2 formulates the problem using spherical polar coordinates, $r, \theta, \phi$. As for the Moffatt problem, linearity and absence of an intrinsic length scale lead to modes for which the velocity components have $r^{\lambda}$ dependence on the radial coordinate, where the exponent $\lambda=\lambda_{r}+i \lambda_{i}$ can be complex. The steady, incompressible Stokes equations and no-slip boundary conditions lead to a homogeneous linear problem which must be satisfied by the $\theta, \phi$ dependent coefficients of the velocity and pressure. The problem only has a non-zero solution for certain discrete values of $\lambda$, thus determining the modes.

In $\S 3$, the Chebyshev-Chebyshev collocation method used to discretize the differential equations is described. This leads to a matrix system of the form $A(\lambda) \chi=0(\chi$ is a column vector formed by the velocity components and pressure at the collocation points), i.e. $\lambda$ must be such that $A(\lambda)$ is singular. Reflection symmetry is employed to split the problem into decoupled symmetric and antisymmetric parts. Thus, there are two families of modes, symmetric and antisymmetric. The methods used to locate the values $\lambda$ and compute the modal velocity field are also described.

Section 4 gives the results. In particular, the modes with the smallest real part of $\lambda$, which decay slowest as $r \rightarrow 0$ and hence should dominate the near-apex flow, are found to have $\lambda=3.26358 \pm 1.16164 \mathrm{i}$. These are referred to as the primary modes and include both symmetric and antisymmetric modes. Secondary modes arise at higher values of $\lambda_{r}$, but, being more rapidly decaying as $r \rightarrow 0$, they are of lesser importance and we have not studied the resulting flows in detail. The most general primary flow which can be constructed is a linear combination of the symmetric and antisymmetric modes. Thus, there is not one, but a two-parameter family of primary flows. As for Moffatt eddies, the velocity decays like $r^{\lambda_{r}}$ as the corner is approached and repeats itself log-periodically at smaller and smaller scales.

Streamlines and particle trajectories are perhaps the most widely used tools for analysing flow structure. Given that the primary flows are steady, streamlines and particle trajectories coincide and are obtained by numerical integration of the equations of particle motion. Three special cases are found, characterized by antisymmetry of the flow under reflection in one of the three symmetry planes of the cone. In these cases, the particle trajectories are time-periodic and hence the streamlines are closed, as for the Moffatt problem. However, for all other flows (i.e. the generic case), they are aperiodic and the streamlines are not closed. Integrating forwards in time, we find that streamlines approach a limiting trajectory which is quasi-periodic and leads to particle motion away from the apex. Likewise, integrating backwards in time, another 


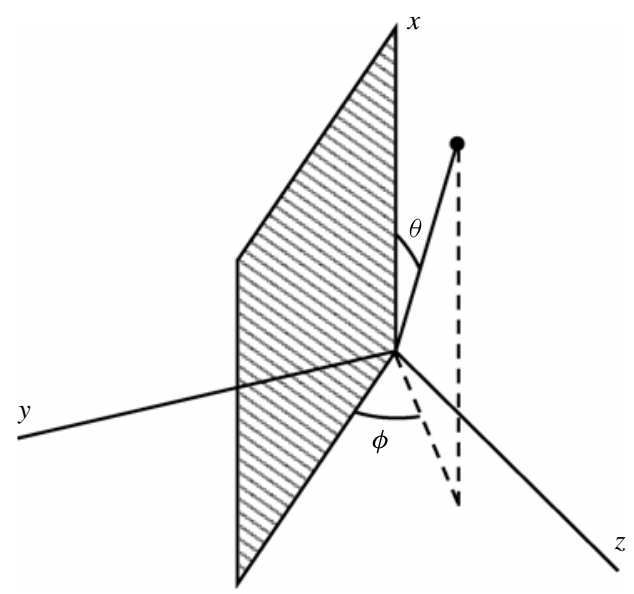

FIgURE 1. The coordinates $\theta$ and $\phi$ (as well as $x, y, z$, which are used in appendix B). The shaded plane, $\phi=0$, is one of three for which the modal problem is symmetric under reflection.

quasi-periodic trajectory is approached for which the particle moves towards the apex. A generic streamline comes in from infinity along the backward-time trajectory, enters a transient phase during which the particle motion is no longer quasi-periodic, before going back out to infinity along the forward-time trajectory.

\section{Formulation}

Using spherical polar coordinates, $r, \theta, \phi$, having origin at the apex, we consider steady, incompressible Stokes flow in the trihedral cone $0 \leqslant \theta \leqslant \pi / 2,|\phi| \leqslant \pi / 4$ (see figure 1). The lack of an intrinsic length scale and linearity of the problem lead to modal solutions of the form

$$
\left(\begin{array}{c}
v_{r} \\
v_{\theta} \\
v_{\phi} \\
\Pi / \mu
\end{array}\right)=r^{\lambda}\left(\begin{array}{c}
u_{r}(\theta, \phi) \\
u_{\theta}(\theta, \phi) \\
u_{\phi}(\theta, \phi) \\
r^{-1} p(\theta, \phi)
\end{array}\right),
$$

where $\boldsymbol{v}, \Pi$ are the velocity and pressure, $\mu$ the dynamic viscosity and $\lambda=\lambda_{r}+\mathrm{i} \lambda_{i}$ a constant which may be complex. As we shall see, only certain discrete values of $\lambda$ permit a non-zero solution. If $\lambda$ is real, the velocity and pressure fields in (2.1) are also real and evidently have power-law dependence on $r$. If $\lambda$ is complex, writing $r^{\lambda}=r^{\lambda_{r}} \exp \left[\mathrm{i} \lambda_{i} \ln r\right]$ shows that the velocity is the product of $r^{\lambda_{r}}$ and a periodic function of $\ln r$ which changes sign every $\pi /\left|\lambda_{i}\right|$. Leaving aside the $r^{\lambda r}$ factor, the velocity field repeats itself $\log$-periodically at smaller and smaller scales. Multiplying $r$ by $\exp \left[-\pi /\left|\lambda_{i}\right|\right]$ flips the sign of the velocity components, while multiplication by $\exp \left[-2 \pi /\left|\lambda_{i}\right|\right]$ leads to repetition. Complex $\lambda$ occur in conjugate pairs, representing modes which are complex conjugates of each other. Physically meaningful (i.e. real) solutions of the governing equations follow from taking the real part of (2.1), a process which combines conjugate modes. Conjugate modes represent the same physical entity; both are needed to express a real flow. 
To avoid divergence of the integrated viscous energy dissipation rate as $r \rightarrow 0$, we require $\lambda_{r}>-1 / 2$. Given this condition, the Reynolds number based on $\boldsymbol{v}$ and $r$ decreases to zero as $r \rightarrow 0$, implying consistency of the Stokes approximation near the apex.

Written in matrix-operator form, the governing equations give

$$
\left(\begin{array}{cccc}
L-2 & -2 \partial_{\theta}-2 \frac{\cos \theta}{\sin \theta} & -\frac{2}{\sin \theta} \partial_{\phi} & 1-\lambda \\
2 \partial_{\theta} & L-\frac{1}{\sin ^{2} \theta} & -2 \frac{\cos \theta}{\sin ^{2} \theta} \partial_{\phi} & -\partial_{\theta} \\
\frac{2}{\sin \theta} \partial_{\phi} & 2 \frac{\cos \theta}{\sin ^{2} \theta} \partial_{\phi} & L-\frac{1}{\sin ^{2} \theta} & -\frac{1}{\sin \theta} \partial_{\phi} \\
\lambda+2 & \partial_{\theta}+\frac{\cos \theta}{\sin \theta} & \frac{1}{\sin \theta} \partial_{\phi} & 0
\end{array}\right)\left(\begin{array}{c}
u_{r} \\
u_{\theta} \\
u_{\phi} \\
p
\end{array}\right)=0
$$

where $\partial_{\theta}, \partial_{\phi}$ are partial derivatives and

$$
L=\partial_{\theta}^{2}+\frac{\cos \theta}{\sin \theta} \partial_{\theta}+\frac{1}{\sin ^{2} \theta} \partial_{\phi}^{2}+\lambda(\lambda+1)
$$

arises from the scalar Laplacian. The first three rows of the matrix operator represent the momentum equation, whereas the last row expresses the incompressibility condition. The no-slip conditions at the walls imply $u_{r}=u_{\theta}=u_{\phi}=0$ for $\theta=0, \pi / 2$ and $\phi= \pm \pi / 4$.

\section{Numerical methods}

The above differential equations are discretized using Chebyshev collocation, with collocation points $\theta=\theta_{n}, \phi=\phi_{l}$, where

$$
\begin{gathered}
\theta_{n}=\frac{1}{4} \pi\left(1-\cos \left(\frac{n \pi}{N_{\theta}+1}\right)\right) \quad 1 \leqslant n \leqslant N_{\theta}, \\
\phi_{l}=\frac{1}{4} \pi \cos \left(\frac{l \pi}{N_{\phi}+1}\right) \quad 1 \leqslant l \leqslant N_{\phi} .
\end{gathered}
$$

The velocity and pressure are represented by their values at the collocation points, organized as column vectors. For instance, $u_{r}^{n l}=u_{r}\left(\theta_{n}, \phi_{l}\right)$ yields an $N_{\theta} N_{\phi}$-dimensional column vector denoted $U_{r}$, with similar definitions for $U_{\theta}, U_{\phi}$ and $P$. Specifically, the order used was $u^{11}, u^{21}, \ldots, u^{N_{\theta} 1}, u^{12}, \ldots, u^{N_{\theta} 2}, \ldots, u^{1 N_{\phi}}, \ldots, u^{N_{\theta} N_{\phi}}$.

Equation (2.2) is evaluated at each of the collocation points, derivatives of the velocity components and pressure being represented by matrix multiplication in the usual manner (see appendix A for details). This leads to the block-matrix system

$$
\left(\begin{array}{cccc}
\Lambda-2 I & -2 \Delta_{\theta}-2 C S & -2 S \Delta_{\phi} & (1-\lambda) I \\
2 \Delta_{\theta} & \Lambda-S^{2} & -2 C S^{2} \Delta_{\phi} & -\tilde{\Delta}_{\theta} \\
2 S \Delta_{\phi} & 2 C S^{2} \Delta_{\phi} & \Lambda-S^{2} & -S \tilde{\Delta}_{\phi} \\
(\lambda+2) I & \Delta_{\theta}+C S & S \Delta_{\phi} & 0
\end{array}\right)\left(\begin{array}{c}
U_{r} \\
U_{\theta} \\
U_{\phi} \\
P
\end{array}\right)=0 .
$$

Within a block, rows correspond to the same equation applied at different collocation points, ordered as for $U_{r}, U_{\theta}, U_{\phi}$ and $P$. The matrices $\Lambda, \Delta_{\theta}$ and $\Delta_{\phi}$ arise from 
the Laplacian and first-order derivatives of the velocity components, $\tilde{\Delta}_{\theta}$ and $\tilde{\Delta}_{\phi}$ come from the pressure derivatives, $C$ and $S$ are matrices representing multiplication by $\cos \theta$ and $1 / \sin \theta$ and $I$ is the unit matrix. Note that these matrices are all $N_{\theta} N_{\phi} \times N_{\theta} N_{\phi}$. Thus, the matrix in (3.3) consists of square blocks of this size. The boundary conditions have been used to express the Laplacian and first-order derivatives of the velocity components and are thus implicit in (3.3).

The final $N_{\theta} N_{\phi}$ rows of (3.3) (incompressibility) imply

$$
U_{r}=E_{\theta} U_{\theta}+E_{\phi} U_{\phi},
$$

where

$$
E_{\theta}=-\frac{1}{\lambda+2}\left(\Delta_{\theta}+C S\right), \quad E_{\phi}=-\frac{1}{\lambda+2} S \Delta_{\phi} .
$$

Using (3.4) to eliminate $U_{r}$ from (3.3),

$$
A\left(\begin{array}{c}
U_{\theta} \\
U_{\phi} \\
P
\end{array}\right)=0
$$

where

$$
A=\left(\begin{array}{ccc}
-2 \Delta_{\theta}-2 C S+(\Lambda-2 I) E_{\theta} & -2 S \Delta_{\phi}+(\Lambda-2 I) E_{\phi} & (1-\lambda) I \\
\Lambda-S^{2}+2 \Delta_{\theta} E_{\theta} & -2 C S^{2} \Delta_{\phi}+2 \Delta_{\theta} E_{\phi} & -\tilde{\Delta}_{\theta} \\
2 C S^{2} \Delta_{\phi}+2 S \Delta_{\phi} E_{\theta} & \Lambda-S^{2}+2 S \Delta_{\phi} E_{\phi} & -S \tilde{\Delta}_{\phi}
\end{array}\right)
$$

consists of $N_{\theta} N_{\phi} \times N_{\theta} N_{\phi}$ blocks.

The original problem, equation (2.2) with zero-velocity boundary conditions, is symmetric under the reflection $\phi \mapsto-\phi$ (see figure 1). This implies modes which are either symmetric $\left(u_{r} \mapsto u_{r}, u_{\theta} \mapsto u_{\theta}, u_{\phi} \mapsto-u_{\phi}, p \mapsto p\right)$ or antisymmetric $\left(u_{r} \mapsto-u_{r}, \quad u_{\theta} \mapsto-u_{\theta}, u_{\phi} \mapsto u_{\phi}, \quad p \mapsto-p\right)$. This property is inherited by the numerical approximation (3.6) and allows splitting of the problem into symmetric and antisymmetric parts, having matrices $A_{s}$ and $A_{a}$. For the sake of simplicity, $N_{\phi}$ is supposed even from here on, hence symmetric and antisymmetric problems with one-half the size of (3.6) and having the form

$$
B(\lambda) X=0,
$$

where $B=A_{s}$ for the symmetric problem and $B=A_{a}$ for the antisymmetric one. The column vector $X$ consists of the first $N_{\theta} N_{\phi} / 2$ elements of $U_{\theta}$, followed by those of $U_{\phi}$ and $P$. The matrices $A_{s}$ and $A_{a}$ inherit the block structure of $A$, with square blocks having $N_{\theta} N_{\phi} / 2$ rows and columns. Given a block of $A$, the corresponding blocks of $A_{s}$ and $A_{a}$ result from dropping the final $N_{\theta} N_{\phi} / 2$ rows and appropriate summation and subtraction of the columns corresponding to $\phi_{l}$ and $-\phi_{l}$.

We want to obtain the values of $\lambda$ for which (3.8) has a non-zero solution (i.e. $B$ is singular) for both the symmetric and antisymmetric problems, since these values lead to modes. To this end, we use Gaussian elimination with partial pivoting to solve

$$
B(\lambda) X(\lambda)=F,
$$


where $F$ is a column vector independent of $\lambda$. As $\lambda$ approaches a value at which $B$ is singular, the quantity

$$
\sigma(\lambda)=\frac{1}{\|X(\lambda)\|},
$$

where $\|$ || denotes the usual norm of a complex vector, will generally tend to zero (it would be an exceedingly unlucky choice of $F$ if this were not true). Thus, we look for solutions of $\sigma(\lambda)=0$. Estimates of such solutions are obtained by a graphical method, described in the next section, and refined using complex Newton-Raphson iteration on the function

$$
f(\lambda)=\frac{1}{F^{H} X(\lambda)},
$$

where $F^{H}$ is the Hermitian conjugate of $F$. The iteration requires the derivative of $f(\lambda)$, which is approximated by the finite difference $(f(\lambda+\varepsilon)-f(\lambda-\varepsilon)) /(2 \varepsilon)$, with $\varepsilon$ a small parameter (we chose $\varepsilon=10^{-4}$ ). Iteration was continued until the difference between successive values of $\lambda$ was less in modulus than a specified tolerance (we used $10^{-12}$ ). Note that double-precision arithmetic (64-bit IEEE 754) was used throughout.

Given a converged value of $\lambda$, solution of (3.9) by Gaussian elimination yields the modal velocity and pressure fields, represented by $X$. Modal symmetry allows calculation of $U_{\theta}$ and $U_{\phi}$ for all collocation points, while (3.4) gives $U_{r}$. Then $u_{r}, u_{\theta}$ and $u_{\phi}$ can be determined at any $\theta$ and $\phi$ using the same polynomial fitting employed in appendix A to express the velocity derivatives (the fitting was implemented by discrete Fourier transforms). Spatial resolution near the corners $\theta=\pi / 2, \phi= \pm \pi / 4$ was augmented using rotational symmetry properties of the modes, as described in appendix B.

\section{Results}

\subsection{Determination of $\lambda$}

Figure 2 shows curves of constant $\sigma(\lambda)$ in the complex $\lambda$-plane for the symmetric (figure $2 a$ ) and antisymmetric (figure $2 b$ ) problems. These results are for $N_{\theta}=N_{\phi}=8$ and $F$ with all elements equal to one (similar results are obtained for other choices of $F$, in particular the zeros of $\sigma(\lambda)$ are insensitive to this choice). Modal values of $\lambda$, obtained using Newton-Raphson with $N_{\theta}=N_{\phi}=16$, are indicated by crosses. There is a zero at $\lambda=1$ in figure $2(a)$, representing the constant pressure, zerovelocity mode, which is of no dynamical significance and is thus ignored. Others occur at $\lambda=3.26358 \pm 1.16164 \mathrm{i}$ for both symmetries and represent modes which, having the smallest $\lambda_{r}$, dominate the flow as $r \rightarrow 0$. These are the primary modes to which we will subsequently focus attention. Owing to numerical discretization, the values of $\lambda$ from the symmetric and antisymmetric problems are not exactly the same. That they should coincide precisely in the absence of numerical errors is a consequence of rotational symmetry and is discussed in appendix B. Their difference is thus a measure of numerical accuracy and, together with a refined calculation using $N_{\theta}=N_{\phi}=32$, indicated that the above values of $\lambda$ are accurate to the given number of decimal places. Secondary modes appear at higher $\lambda_{r}$. Those below $\lambda_{r}=7$ lie at $\lambda=5.294 \pm 1.645 \mathrm{i}(\mathrm{s} / \mathrm{a}), \lambda=5.354 \pm 1.287 \mathrm{i}(\mathrm{s})$ and $\lambda=5.381$ (a), where (s) indicates a symmetric mode, (a) an antisymmetric mode and (s/a) implies both symmetries for the same value of $\lambda$. We restrict attention to the primary modes from here on. 

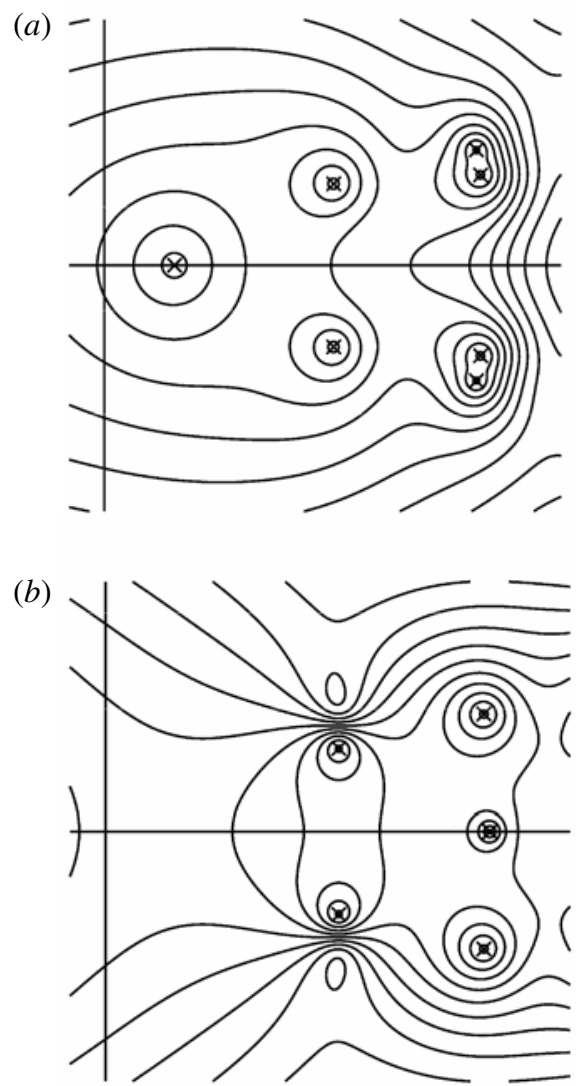

FIgURE 2. Contours of constant $\sigma(\lambda)$ in the complex $\lambda$-plane for $(a)$ the symmetric problem, (b) the antisymmetric problem $\left(N_{\theta}=N_{\phi}=8,-0.5 \leqslant \lambda_{r} \leqslant 6.5,\left|\lambda_{i}\right| \leqslant 3.5\right)$. The crosses represent zeros obtained using Newton-Raphson iteration with $N_{\theta}=N_{\phi}=16$.

\subsection{Velocity fields and particle trajectories of the primary flows}

Combining the two primary modes with $\lambda=3.26358+1.16164 \mathrm{i}$ and taking the real part, the velocity gives

$$
\boldsymbol{v}=\operatorname{Re}\left\{r^{\lambda}\left(\zeta \boldsymbol{u}^{s}(\theta, \phi)+(1-\zeta) \mathrm{e}^{\mathrm{i} \psi} \boldsymbol{u}^{a}(\theta, \phi)\right)\right\},
$$

where $\operatorname{Re}\{\}$ denotes the real part of a complex quantity, $\boldsymbol{u}^{s}$ and $\boldsymbol{u}^{a}$ represent the symmetric and antisymmetric modes and $0 \leqslant \zeta \leqslant 1,0 \leqslant \psi<2 \pi$ parameterize the family of flows given by (4.1). For definiteness sake, $\boldsymbol{u}^{s}$ and $\boldsymbol{u}^{a}$ are normalized by requiring $u_{\theta}^{s}=1$ and $u_{\phi}^{a}=1$ on the cone axis, $\theta=\arctan (\sqrt{2}), \phi=0$. The parameter $\zeta$ expresses the proportion of the symmetric component in the symmetric-antisymmetric mix, while $\psi$ determines the relative phases of the two components. A completely general combination would multiply by an arbitrary complex constant, prior to taking the real part in (4.1). This is equivalent to rescaling $r$ and multiplying $\boldsymbol{v}$ by a real constant. Thus, to within a change of radial scale and a multiplicative constant, equation (4.1) provides the most general form of the velocity field resulting from $\lambda=3.26358+1.16164 \mathrm{i}$. The rest of this article concerns the particle trajectories resulting from (4.1). Note that $\boldsymbol{v}$ changes sign and is divided by $\exp \left[\pi \lambda_{r} / \lambda_{i}\right]=6810$ 


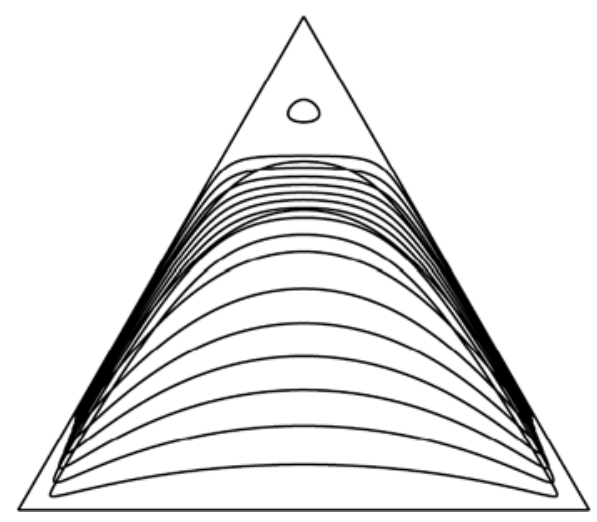

FIGURE 3. Radial projection of 10 streamlines of the purely antisymmetric primary flow $(\zeta=0)$ onto a plane perpendicular to the cone axis. Each point within the triangle represents a particular $(\theta, \phi)$ with the summit corresponding to $\theta=0$, the base to $\theta=\pi / 2$, the left-hand side to $\phi=-\pi / 4$ and the right-hand side to $\phi=\pi / 4$. These results were obtained using $\psi=0$ and the initial conditions $\rho=\phi=0$ and $\theta=\pi / 22,2 \pi / 22, \ldots, 10 \pi / 22$.

when $r$ is reduced by a factor of $\exp \left[\pi / \lambda_{i}\right]=14.946$. A radial period of the flow corresponds to a reduction of $r$ by a factor of $\exp \left[2 \pi / \lambda_{i}\right]=223.38$.

Particle trajectories (also known as streamlines since the flow is steady) were determined by fourth-order Runge-Kutta integration of

$$
\frac{\mathrm{d}}{\mathrm{d} \tau}\left(\begin{array}{c}
\rho \\
\theta \\
\phi
\end{array}\right)=\left(V_{\rho}^{2}+V_{\theta}^{2}+V_{\phi}^{2}\right)^{-1 / 2}\left(\begin{array}{c}
V_{\rho} \\
V_{\theta} \\
V_{\phi}
\end{array}\right),
$$

where $\rho=\ln r, \tau$ is a time-like variable defined by

$$
\frac{\mathrm{d} \tau}{\mathrm{d} t}=r^{\lambda_{r}-1}\left(V_{\rho}^{2}+V_{\theta}^{2}+V_{\phi}^{2}\right)^{1 / 2}
$$

and

$$
\begin{gathered}
V_{\rho}=\operatorname{Re}\left\{\exp \left[\mathrm{i} \lambda_{i} \rho\right]\left(\zeta u_{r}^{s}(\theta, \phi)+(1-\zeta) \mathrm{e}^{\mathrm{i} \psi} u_{r}^{a}(\theta, \phi)\right)\right\}, \\
V_{\theta}=\operatorname{Re}\left\{\exp \left[\mathrm{i} \lambda_{i} \rho\right]\left(\zeta u_{\theta}^{s}(\theta, \phi)+(1-\zeta) \mathrm{e}^{\mathrm{i} \psi} u_{\theta}^{a}(\theta, \phi)\right)\right\}, \\
V_{\phi}=\frac{1}{\sin \theta} \operatorname{Re}\left\{\exp \left[\mathrm{i} \lambda_{i} \rho\right]\left(\zeta u_{\phi}^{s}(\theta, \phi)+(1-\zeta) \mathrm{e}^{\mathrm{i} \psi} u_{\phi}^{a}(\theta, \phi)\right)\right\} .
\end{gathered}
$$

Given the two-parameter nature of the flow and the three-dimensional space of initial conditions, complete characterization of the trajectories is not feasible and we describe results of a limited (but large, more than 14000 trajectories in all) number of calculations. The values $N_{\theta}=N_{\phi}=32$ and a $\tau$-step of $10^{-2}$ were used throughout.

When $\zeta=0$ the flow is purely antisymmetric and (performing 1000 trajectory calculations) we found periodic trajectories, hence closed streamlines, for all initial conditions $(\rho(\tau), \theta(\tau)$ and $\phi(\tau)$ are all periodic with the same period). Figure 3 shows the radial projection of a few streamlines onto a plane perpendicular to the cone axis. Trajectories which cross the plane $\phi=0$ and return there are obliged by antisymmetry to repeat (with a change in sign of $\phi$ ) their previous time evolution in reverse, thus producing closed streamlines with mirror symmetry. Rotation about the 


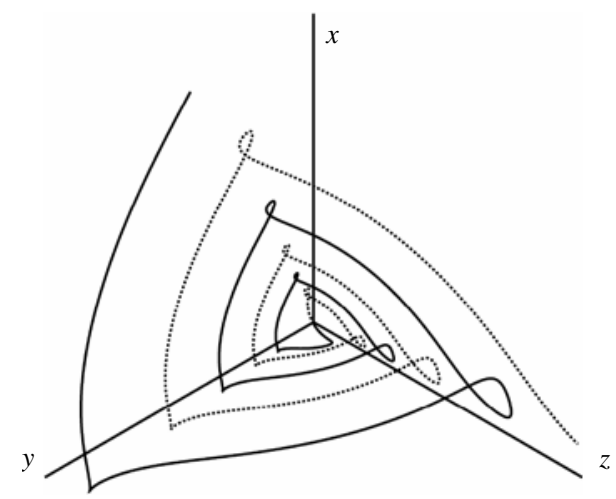

FIGURE 4. Streamline of the flow $\zeta=0.5, \psi=1$ obtained by forward- and backward-time integration of the particle trajectory equations with the initial conditions $\rho=\phi=0$ and $\theta=1$. An orthogonal projection of $(X, Y, Z)$ onto a plane of constant $X+Y+Z$ is shown (see the main text for details). The solid and dashed curves represent the forward- and backward-time parts of the streamline, respectively. Particles come in from infinity along the dashed curve and are thrown back out along the solid curve.

cone axis by angles $\pm 2 \pi / 3$ (cf. appendix B) yields two other flows of periodic type $(\zeta=\sqrt{3} /(\sqrt{3}+1)$ and $\psi=0, \pi)$.

In all other flows, the computed trajectories (13750 in all) were aperiodic. Figure 4 illustrates a particular case. In order to accommodate the large range of $r$ (recall that a radial period corresponds to a factor of 223.38), the modified radial coordinate $r^{1 / 6}$ is used. More precisely, the particle location is represented by $(X, Y, Z)$, where $X=r^{1 / 6} \cos \theta, \quad Y=r^{1 / 6} \sin \theta \cos (\phi+\pi / 4)$ and $Z=r^{1 / 6} \sin \theta \sin (\phi+\pi / 4)$, and is orthogonally projected onto a plane of constant $X+Y+Z$. The figure covers the range $0<X, Y, Z<10$ (thus encompassing $r$ up to above $10^{6}$ ). Use of $r^{1 / 6}$ means that the figure is not a photographic representation of the streamline, but it nonetheless conveys its overall character. Particles spiral in, reach a minimum $r$, then spiral back out again.

Although figure 4 is more immediately assimilated, the radial projection and plot of $\rho(\tau)$ in figure 5 make it apparent that, although the trajectory is aperiodic, it approaches a quasi-periodic limit at large $\tau$. By quasi-periodic we mean that $\theta(\tau)$ and $\phi(\tau)$ are periodic, while $\rho(\tau)$ is the sum of linear and periodic functions. The resulting $\rho(\tau)$ increases by $2 \pi / \lambda_{i}$ per quasi-period. We refer to this quasiperiodic limit trajectory as 'forward-time' to distinguish it from another quasi-periodic trajectory which is approached when running the calculation backwards in time. The backward-time trajectory can be obtained from the forward-time trajectory by adding $\pi / \lambda_{i}$ to $\rho$ and switching the sign of $\tau$. Hence, forward- and backward-time trajectories have the same quasi-period and the same projection onto the coordinates $(\theta, \phi)$. They appear to be unique (up to addition of an arbitrary constant to $\tau$ and subject to the exceptional cases discussed below) for a given flow and to be approached from generic initial conditions. Thus, we expect a generic trajectory to be close to the backward-time trajectory as $\tau \rightarrow-\infty$, followed by a transient phase in which it is no longer quasi-periodic, before approaching the forward-time trajectory as $\tau \rightarrow+\infty$ and becoming quasi-periodic once more. Although, as illustrated by figure $5(b)$, the forward-time trajectory may have intervals of time during which $\rho(\tau)$ decreases, it increases overall. Likewise, the backward-time trajectory implies overall particle 
(a)

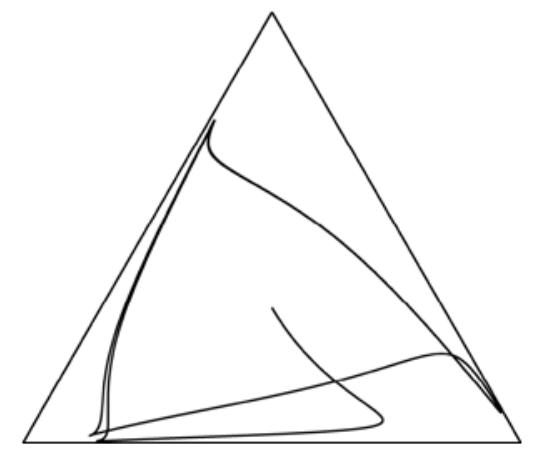

(b)

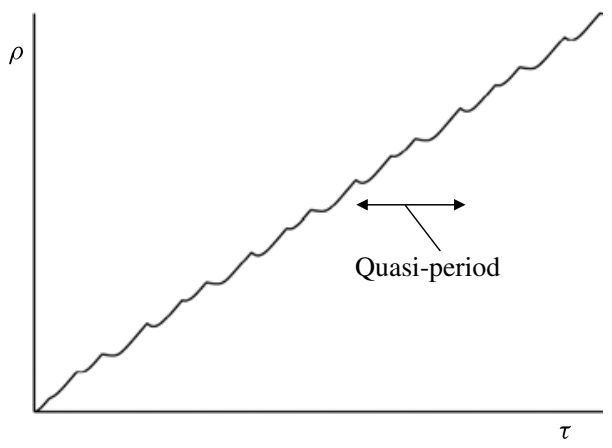

FIGURE 5. Trajectory of the flow $\zeta=0.5, \psi=1$ with initial conditions $\rho=\phi=0$ and $\theta=1:(a)$ radial projection as in figure $3 ;(b)$ plot of $\rho(\tau)$.

motion towards the cone apex. A generic streamline will spiral in towards the apex, then be thrown out again following the transient phase. Of course, if a particle lies precisely on one of the limit trajectories, it will move progressively away from or towards the apex. However, such behaviour only concerns the subspace of dimension one consisting of the two limit trajectories and is thus far from generic.

Exceptions to the statement made above that the limit trajectories are unique occur for the purely symmetric flow, $\zeta=1$, and its rotated counterparts, $\zeta=1 /(\sqrt{3}+1)$, $\psi=0, \pi$. In these cases, the symmetry plane forms an impenetrable barrier for fluid particles and there are two forward- and backward-time trajectories, related by mirror symmetry (cf. figure $9 j$ ). This does not affect the conclusion concerning a generic particle; it simply adopts the limit trajectories on its side of the barrier as $\tau \rightarrow \pm \infty$.

Figures 6 and 7 illustrate the behaviour close to the periodic case, while figure 8 eliminates the transient phase to show the final quasi-periodic trajectory for the same flow. Figure 9 shows the quasi-periodic trajectories of a variety of primary flows.

\subsection{Limit trajectories as functions of real time}

Up to now, we have used the time-like variable $\tau$. The special cases in which the trajectories are periodic in $\tau$ also lead to periodicity in the 'real' time, $t$. However, for other flows it may be of interest to examine what quasi-periodicity in $\tau$ of the limit trajectories implies for particle motion as a function of $t$.

The relation, (4.3), between $t$ and $\tau$ can be written as

$$
\frac{\mathrm{d} t}{\mathrm{~d} \tau}=\left(V_{\rho}^{2}+V_{\theta}^{2}+V_{\phi}^{2}\right)^{-1 / 2} \exp \left[\left(1-\lambda_{r}\right) \rho\right]
$$




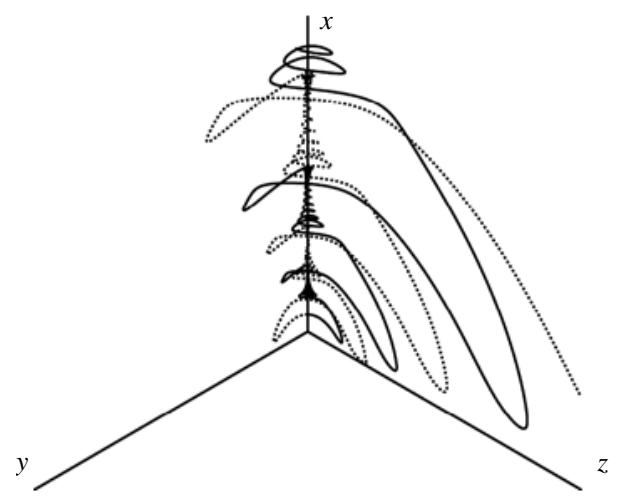

FIgURE 6. As figure 4, but for the flow $\zeta=0.01, \psi=0$ and the initial conditions $\rho=\phi=0$ and $\theta=0.5$.

(a)

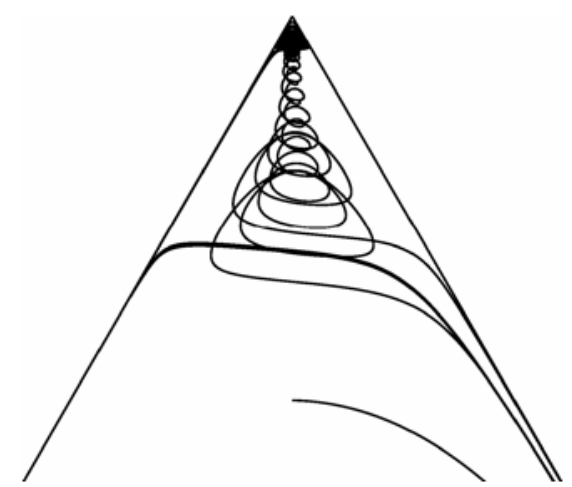

(b)

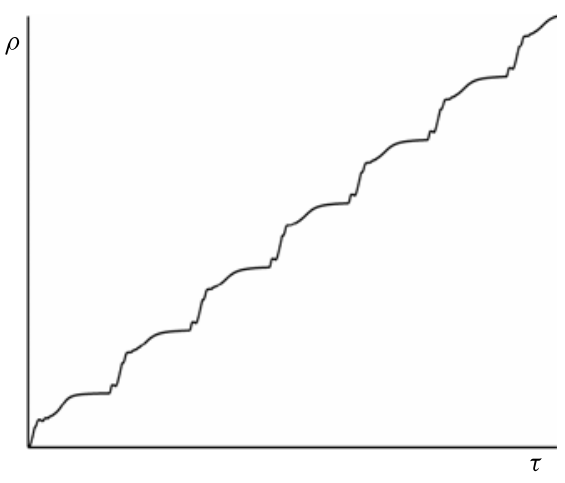

FIGURE 7. Trajectory of the flow $\zeta=0.01, \psi=0$ with initial conditions $\rho=\phi=0$ and $\theta=0.5$ : (a) radial projection as in figure 3 (only the top half is shown); $(b)$ plot of $\rho(\tau)$.

Consider the forward-time trajectory and let $\tau_{n}$ be such that $\tau_{n+1}-\tau_{n}$ is a quasi-period. Denoting the corresponding times by $t_{n}$, integration of (4.7) gives

$$
t_{n+1}-t_{n}=\int_{\tau_{n}}^{\tau_{n+1}}\left(V_{\rho}^{2}+V_{\theta}^{2}+V_{\phi}^{2}\right)^{-1 / 2} \exp \left[\left(1-\lambda_{r}\right) \rho\right] \mathrm{d} \tau .
$$

Quasi-periodicity implies increase of $\rho(\tau)$ by $2 \pi / \lambda_{i}$ every quasi-period, hence periodicity of $\left(V_{\rho}^{2}+V_{\theta}^{2}+V_{\phi}^{2}\right)^{-1 / 2}$ and

$$
t_{n+1}-t_{n}=\left(t_{1}-t_{0}\right) \exp \left[2 \pi \frac{1-\lambda_{r}}{\lambda_{i}} n\right]
$$

according to (4.8). The solution of (4.9) is

$$
t_{n}=t_{\infty}+\frac{t_{1}-t_{0}}{\exp \left[2 \pi\left(\left(1-\lambda_{r}\right) / \lambda_{i}\right)\right]-1} \exp \left[2 \pi \frac{1-\lambda_{r}}{\lambda_{i}} n\right],
$$

where $t_{\infty}$ is some constant. Because $\lambda_{r}>1, t_{n} \rightarrow t_{\infty}$ from below as $n \rightarrow \infty$, the equally spaced $\tau_{n}$ becoming a geometrically convergent sequence in $t$. It follows that $\rho \rightarrow \infty$ at the finite time, $t_{\infty}$. Likewise, the backward-time trajectory originates 


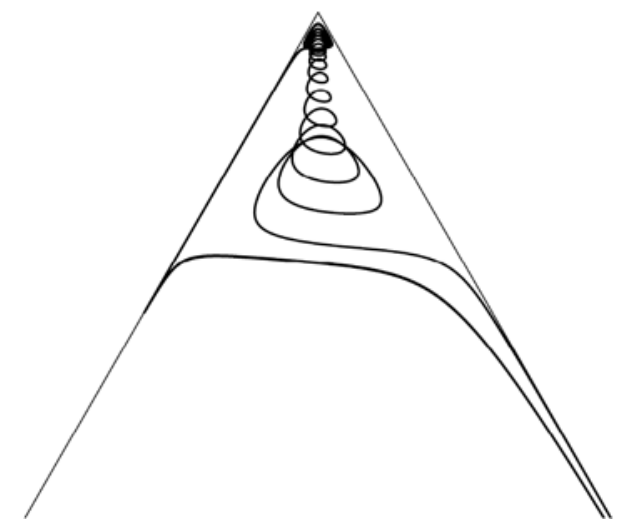

FIGURE 8. Quasi-periodic trajectory of the flow $\zeta=0.01, \psi=0$ Radial projection as for figure $7(a)$. The spiralling part of the curve corresponds to an attempt by the particle at approximately periodic behaviour, an attempt which fails due to interaction with the corner $\theta=0$ and the side walls $\phi= \pm \pi / 4$.

from infinity at finite time. Thus, a generic trajectory comes from, and goes back to, infinity at finite times. It should, of course, be borne in mind that use of the primary modes of Stokes flow in an infinite cone to represent real flows is only justified near the apex.

Finally, in the case of the forward-time trajectory, it can be shown, using (4.10), that $\theta$ and $\phi$ are periodic functions, and $\rho$ a quasi-periodic function, of $\ln \left(t_{\infty}-t\right)$ with (quasi-)period $2 \pi\left(\lambda_{r}-1\right) / \lambda_{i}$. A similar statement holds for the backward-time trajectory. Thus, quasi-periodicity with respect to $\tau$ translates to quasi-periodicity as a function of $\ln \left|t-t_{\infty}\right|$, where $t_{\infty}$ is the time at which the forward- or backward-time trajectory goes out to or comes in from infinity.

\section{Conclusions}

Moffatt-type modes in an infinite trihedral cone bounded by three orthogonal planes have been derived. The modal velocity has complex power-law dependence, $r^{\lambda}$, on the radial coordinate in a spherical polar coordinate system, $r, \theta, \phi$. Following spatial discretization using Chebyshev-Chebyshev collocation in $\theta$ and $\phi$ and decomposition into symmetric and antisymmetric parts, the exponent $\lambda$ is determined by the requirement of a non-zero solution of one or other of the matrix problems $A_{s}(\lambda) X=0$ or $A_{a}(\lambda) X=0$, where the subscripts $s$ and $a$ refer to symmetric and antisymmetric modes.

Symmetric and antisymmetric primary modes are found to have the same values of $\lambda$, namely $\lambda=3.26358 \pm 1.16164 \mathrm{i}$, with secondary modes at higher $\lambda_{r}$. Primary flows form a two-parameter family obtained by linear combination of the symmetric and antisymmetric modes. Their trajectories are time-periodic, implying closed streamlines, in the special cases for which the flow is antisymmetric with respect to one of the symmetry planes of the cone. In all other cases, aperiodic trajectories are found. They then approach quasi-periodic limits when the trajectory equations are integrated either forwards or backwards in time. A generic particle spirals in from infinity following the backward-time trajectory, undergoes a transient phase in which the motion is no longer quasi-periodic, and is then thrown back out to infinity along the forward-time 
(a)

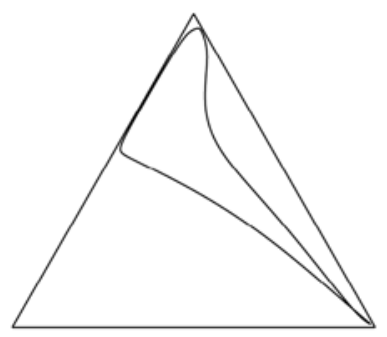

(c)

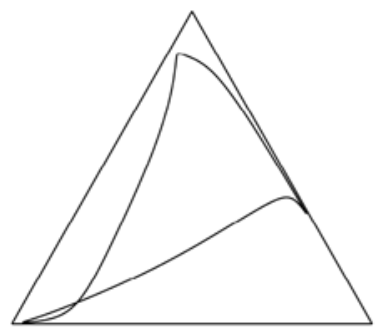

(e)

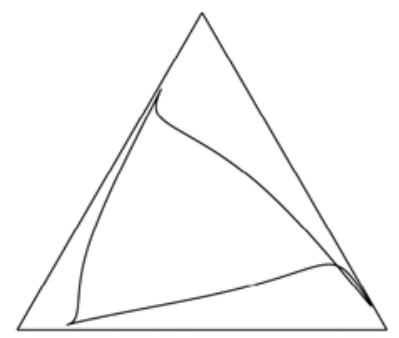

$(g)$

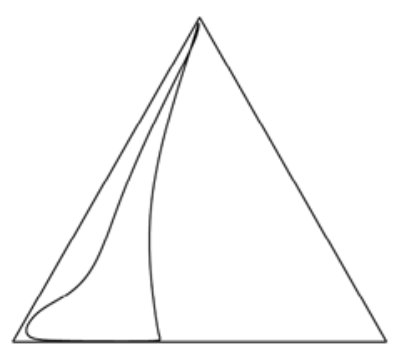

(i)

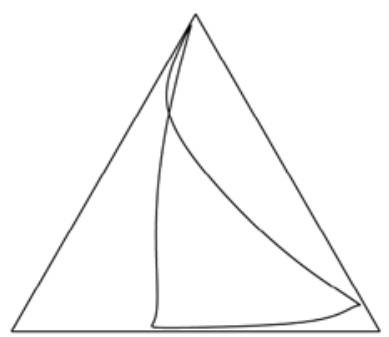

(b)

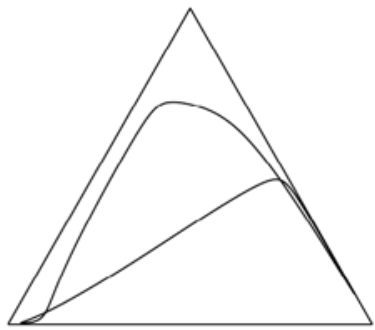

(d)

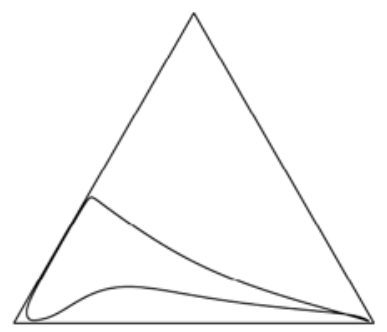

$(f)$

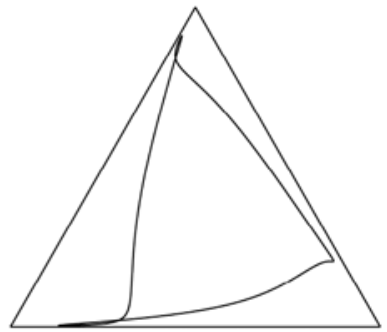

(h)

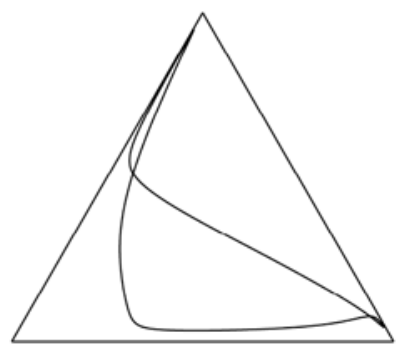

$(j)$

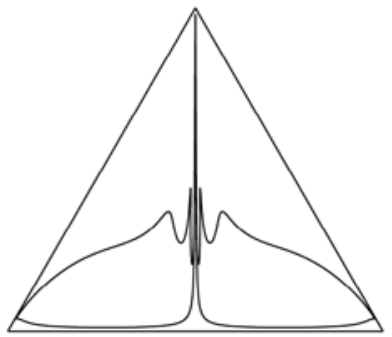

FIGURE 9. Quasi-periodic trajectories of the flows $(a-c) \zeta=1 / 4,(d-f) \zeta=1 / 2,(g-i)$ $\zeta=3 / 4$ and $(j) \zeta=1$. Radial projection as for figure 3 . Within each triplet, $\psi$ takes the values $0, \pi / 3$ and $2 \pi / 3$. Note that $\psi \mapsto \psi+\pi, \phi \mapsto-\phi$ leaves trajectories unchanged, hence there is no need to consider $\psi \geqslant \pi$. Recall that the radial projections of the forwardand backward-time trajectories coincide (these trajectories differ in $\rho$ and in the direction of motion of the particle around the above curves). 
trajectory. Such a flow can hardly be described as consisting of eddies, this term having, as it does, the connotation of localized regions of flow recirculation.

Finally, it would be interesting to make comparisons with the near-corner results of the numerical computations of Leriche \& Labrosse (2011), who studied Stokes modes in a cube. It might also be interesting to extend the analysis to trihedral cones with non-orthogonal planes.

\section{Appendix A. Chebyshev derivative matrices}

Let $f(x)$ be a function on $|x| \leqslant 1$ taking the values $f\left(x_{i}\right)=f_{i}$ at

$$
x_{i}=\cos \left(\frac{\pi i}{N+1}\right) \quad 0 \leqslant i \leqslant N+1 .
$$

As is well-known, the derivative of $f(x)$ can be approximated by that of the $(N+1)$ th degree polynomial which matches $f\left(x_{i}\right)=f_{i}$, leading to

$$
f^{\prime}\left(x_{i}\right) \sim \sum_{j=0}^{N+1} D_{i j}^{N} f_{j} \quad 0 \leqslant i \leqslant N+1,
$$

where $D^{N}$ is the $(N+2) \times(N+2)$ matrix given by

$$
\begin{gathered}
D_{i j}^{N}=\frac{c_{i}}{c_{j}} \frac{(-1)^{i+j}}{x_{i}-x_{j}} \quad i \neq j, \quad 0 \leqslant i, j \leqslant N+1, \\
D_{i i}^{N}=\frac{1}{2} \frac{x_{i}}{x_{i}^{2}-1} \quad 1 \leqslant i \leqslant N, \\
D_{00}^{N}=-D_{N+1, N+1}^{N}=\frac{1}{6}\left(2(N+1)^{2}+1\right),
\end{gathered}
$$

in which

$$
c_{i}= \begin{cases}1 & 1 \leqslant i \leqslant N \\ 2 & i=0, N+1 .\end{cases}
$$

Applying (A 2) with $x=1-4 \theta / \pi, N=N_{\theta}$ and $x=4 \phi / \pi, N=N_{\phi}$ yields

$$
D_{\theta}=-\frac{4}{\pi} D^{N_{\theta}}, \quad D_{\phi}=\frac{4}{\pi} D^{N_{\phi}}
$$

for the derivative matrices with respect to $\theta$ and $\phi$. Taking $f=u_{r}$ and using the conditions of zero velocity at the boundaries gives

$$
\partial_{\theta} u_{r} \mapsto \Delta_{\theta} U_{r}, \quad \partial_{\phi} u_{r} \mapsto \Delta_{\phi} U_{r}, \quad L u_{r} \mapsto \Lambda U_{r}
$$

for the vectors of values of the derivatives and Laplacian of $u_{r}$ at the collocation points $\theta=\theta_{n}, \phi=\phi_{l}\left(1 \leqslant n \leqslant N_{\theta}, 1 \leqslant l \leqslant N_{\phi}\right)$, ordered as for $U_{r}, U_{\theta}, U_{\phi}$ and $P$. The $N_{\theta} N_{\phi} \times N_{\theta} N_{\phi}$ matrices $\Delta_{\theta}, \Delta_{\phi}$ and $\Lambda$ follow from

$$
\begin{gathered}
\left(\Delta_{\theta} U_{r}\right)^{n l}=\sum_{m=1}^{N_{\theta}}\left(D_{\theta}\right)_{n m} u_{r}^{m l}, \quad\left(\Delta_{\phi} U_{r}\right)^{n l}=\sum_{k=1}^{N_{\phi}}\left(D_{\phi}\right)_{l k} u_{r}^{n k}, \\
\left(\Lambda U_{r}\right)^{n l}=\sum_{m=1}^{N_{\theta}}\left(D_{\theta}^{2}\right)_{n m} u_{r}^{m l}+\frac{\cos \theta_{n}}{\sin \theta_{n}} \sum_{m=1}^{N_{\theta}}\left(D_{\theta}\right)_{n m} u_{r}^{m l}+\frac{1}{\sin ^{2} \theta_{n}} \sum_{k=1}^{N_{\phi}}\left(D_{\phi}^{2}\right)_{l k} u_{r}^{n k}+\lambda(\lambda+1) u_{r}^{n l} .
\end{gathered}
$$


Replacing $r$ by $\theta$ or $\phi$ in (A 8) gives expressions for the derivatives and Laplacian of the other velocity components.

The pressure derivatives are determined by a slightly different method. The $(N-1)$ thdegree polynomial matching $f\left(x_{i}\right)=f_{i}$ for $1 \leqslant i \leqslant N$ (but not for $i=0, N+1$ ) is differentiated to obtain

$$
f^{\prime}\left(x_{i}\right) \sim \sum_{j=1}^{N} \tilde{D}_{i j}^{N} f_{j} \quad 1 \leqslant i \leqslant N,
$$

where $\tilde{D}^{N}$ is the $N \times N$ matrix given by

$$
\tilde{D}_{i j}^{N}=\frac{1}{1-x_{i}^{2}}\left(\left(1-x_{j}^{2}\right) D_{i j}^{N}+2 x_{i} \delta_{i j}\right) \quad 1 \leqslant i, j \leqslant N
$$

and $\delta_{i j}=0$ for $i \neq j$ and $\delta_{i i}=1$. This leads to the derivative matrices

$$
\tilde{D}_{\theta}=-\frac{4}{\pi} \tilde{D}^{N_{\theta}}, \quad \tilde{D}_{\phi}=\frac{4}{\pi} \tilde{D}^{N_{\phi}},
$$

and, hence, taking $f=p$,

$$
\partial_{\theta} p \mapsto \tilde{\Delta}_{\theta} P, \quad \partial_{\phi} p \mapsto \tilde{\Delta}_{\phi} P,
$$

where the $N_{\theta} N_{\phi} \times N_{\theta} N_{\phi}$ matrices $\tilde{\Delta}_{\theta}$ and $\tilde{\Delta}_{\phi}$ follow from

$$
\left(\tilde{\Delta}_{\theta} P\right)^{n l}=\sum_{m=1}^{N_{\theta}}\left(\tilde{D}_{\theta}\right)_{n m} p^{m l}, \quad\left(\tilde{\Delta}_{\phi} P\right)^{n l}=\sum_{k=1}^{N_{\phi}}\left(\tilde{D}_{\phi}\right)_{l k} p^{n k} .
$$

Note that the above procedure avoids introducing values of the pressure on the boundary.

\section{Appendix B. Consequences of rotational symmetry}

As well as reflection symmetry, the modal problem is invariant under rotation about the cone axis, $\theta=\arctan (\sqrt{2}), \phi=0$, by an angle $2 \pi / 3$. The effect of such rotation is simplest to describe using the Cartesian coordinates $x=r \cos \theta, y=r \sin \theta \cos (\phi+\pi / 4)$ and $z=r \sin \theta \sin (\phi+\pi / 4)$ (see figure 1 ). Given velocity and pressure fields $\boldsymbol{u}(x, y, z)$ and $p(x, y, z)$, the flow following rotation has velocity $\hat{\boldsymbol{u}}(x, y, z)$ and pressure $\hat{p}(x, y, z)$, where $\hat{p}(x, y, z)=p(y, z, x)$ and

$$
\hat{u}_{x}(x, y, z)=u_{z}(y, z, x), \quad \hat{u}_{y}(x, y, z)=u_{x}(y, z, x), \quad \hat{u}_{z}(x, y, z)=u_{y}(y, z, x) .
$$

We write (B 1) symbolically as $\hat{\boldsymbol{u}}=R \boldsymbol{u}$, so $R$ is an operator expressing the effect of rotation on the velocity field. Since the modal problem is invariant under rotation, if $\boldsymbol{u}$ and $p$ are a solution with exponent $\lambda, R \boldsymbol{u}$ and $\hat{p}$ are also a solution with the same $\lambda$.

Applying this result to the primary modes, $R \boldsymbol{u}^{s}$ and $R \boldsymbol{u}^{a}$ are also primary modes, hence they can each be expressed as a linear combination of $\boldsymbol{u}^{s}$ and $\boldsymbol{u}^{a}$. Given the normalization defined in the main text, the coefficients can be determined by considering the effect of rotation for $u_{\theta}$ and $u_{\phi}$ on the cone axis, leading to

$$
R \boldsymbol{u}^{s}=\frac{1}{2}\left(\sqrt{3} \boldsymbol{u}^{a}-\boldsymbol{u}^{s}\right), \quad R \boldsymbol{u}^{a}=-\frac{1}{2}\left(\sqrt{3} \boldsymbol{u}^{s}+\boldsymbol{u}^{a}\right) .
$$

Equation (B 2) implies

$$
\boldsymbol{u}^{s}=-\frac{1}{2}\left(\sqrt{3} R \boldsymbol{u}^{a}+R \boldsymbol{u}^{s}\right), \quad \boldsymbol{u}^{a}=\frac{1}{2}\left(\sqrt{3} R \boldsymbol{u}^{s}-R \boldsymbol{u}^{a}\right),
$$




$$
\boldsymbol{u}^{s}=\frac{1}{2}\left(\sqrt{3} R^{-1} \boldsymbol{u}^{a}-R^{-1} \boldsymbol{u}^{s}\right), \quad \boldsymbol{u}^{a}=-\frac{1}{2}\left(\sqrt{3} R^{-1} \boldsymbol{u}^{s}+R^{-1} \boldsymbol{u}^{a}\right),
$$

where $R^{-1}$ is the inverse of $R$, i.e. rotation of the velocity field in the opposite sense. The above relations should hold for the exact modal velocity fields and approximately for the numerically determined ones. They can be employed to assess numerical accuracy of $\boldsymbol{u}^{s}$ and $\boldsymbol{u}^{a}$. Using $N_{\theta}=N_{\phi}=32$, we found a maximum departure from (B 3) and (B 4) of $8 \times 10^{-6}$ for $u_{r}$ and $4 \times 10^{-7}$ for $u_{\theta}$ and $u_{\phi}$.

Equations (B 3) and (B 4) were also employed to augment the spatial resolution near the corners $\theta=\pi / 2, \phi= \pm \pi / 4$. First divide the flow into three regions: (i) $x \geqslant y$, $x \geqslant z$; (ii) $x<y, y \geqslant z$; and (iii) $x<z, y<z$. No use is made of (B 3) or (B 4) in region 1, while (B 3) is employed in region (ii) and (B 4) in region (iii). Thus, rotation symmetry allows the determination of the velocity in regions (ii) and (iii) in terms of that in region (i), where the spatial resolution near the corner $\theta=0$ is much better.

Rotational symmetry leads to three independent families of modes (in general, neither symmetric nor antisymmetric) such that

$$
\boldsymbol{R} \boldsymbol{u}=\mathrm{e}^{\mathrm{i} 2 \pi k / 3} \boldsymbol{u},
$$

where $k=0, \pm 1$. Reflection symmetry implies that the families $k= \pm 1$ have the same values of $\lambda$. If $\lambda$ results from $k= \pm 1$, as is found to be the case for the primary modes, there are two independent solutions with that value of $\lambda$. Thus, the space of primary mode solutions is of dimension two, which is the reason why there are both symmetric and antisymmetric primary modes. The secondary exponents $\lambda=5.294 \pm 1.645 \mathrm{i}$ also arise from $k= \pm 1$ and, hence, include both symmetric and antisymmetric modes. However, $\lambda=5.354 \pm 1.287 \mathrm{i}$ (s) and $\lambda=5.381$ (a) come from $k=0$ and do not encompass both symmetries.

\section{REFERENCES}

Gomilko, A. M., Malyuga, V. S. \& Meleshio, V. V. 2003 On steady Stokes flow in a trihedral rectangular corner. J. Fluid Mech. 476, 159-177.

Hall, O., Hills, C. P. \& Gilbert, A. D. 2009 Non-axisymmetric Stokes flow between concentric cones. Q. J. Mech. Appl. Maths 62 (2), 131-148.

Hills, C. P. \& Moffatt, H. K. 2000 Rotary honing: a variant of the Taylor paint-scraper problem. J. Fluid Mech. 418, 119-135.

Leriche, E. \& LABrosse, G. 2011 Are there localized eddies in the trihedral corners of the Stokes eigenmodes in cubical cavity? Comput. Fluids 43 (1), 98-101.

LiU, C. H. \& JosePH, D. D. 1978 Stokes flow in conical trenches. SIAM J. Appl. Maths 34, 286-296.

Malhotra, C. P., Weidman, P. D. \& Davis, A. M. J. 2005 Nested toroidal vortices between concentric cones. J. Fluid Mech. 522, 117-139.

Malyuga, V. S. 2005 Viscous eddies in a circular cone. J. Fluid Mech. 522, 101-116.

Moffatt, H. K. 1964 Viscous and resistive eddies near a sharp corner. J. Fluid Mech. 18, 1-18.

Moffatt, H. K. \& MAK, V. 1998 Corner singularities in three-dimensional Stokes flow. In Nonlinear Singularities in Deformation and Flow (ed. D. Durban \& J. R. A. Pearson), pp. 21-26. Kluwer.

Shankar, P. N. 2005 Moffatt eddies in the cone. J. Fluid Mech. 539, 113-135.

ShanKar, P. N. 2007 Slow Viscous Flows. Imperial College Press.

WAKIYA, S. 1976 Axisymmetric flow of a viscous fluid near the vertex of a body. J. Fluid Mech. 78, 737-747. 